\title{
Application Research of Cloud Computing in the Smart Grid Information Processing
}

\author{
Di Mou, Xuanxuan Tian, Tianshu Zhang, Jinwei Zhang, Zhenhua Li and Chenjun He \\ School of Three Gorges University, Yichang, 443000, China
}

\begin{abstract}
With the rapid development of national economy, our demand for power resources has also increased rapidly. The traditional power grid can not be achieved operational status of equipment for effective monitoring and control, we will not be able to ensure the quality and quality of electricity service. Cloudbased build smart grid information platform enables gathering the parameter of grid operation state, greatly increasing the economic efficiency of power enterprises. Firstly, cloud computing and smart grid briefly described, and then pointed out that cloud computing applications in the smart grid information platform, and specialized of cloud-based smart grid information platform, in order for the power grid enterprises to enhance their level of service and provide effective reference.
\end{abstract}

KEYWORD: cloud computing; smart power grids; information platform; Application

\section{INTRODUCTION}

Traditional network monitoring and diagnostic techniques have been unable to meet the needs of China's rapid economic development, grid maintenance, management and promotion is becoming increasingly difficult, and the grid monitoring data can not be shared in real time issue further hampered achieve grid monitoring panoramic goals (Zhiyong $\mathrm{Xu}$, 2013). As cloud computing technology continues to develop, build their cloud-based computing technology smart grid information platform can effectively meet the huge demand for information storage and management of smart grid for power companies to automate information and provide a huge target grid security.

\section{CLOUD COMPUTING AND SMART GRID} DEVELOPMENT SITUATION

The so-called smart grid refers to the more traditional grid intelligence, information grid pattern, which has outstanding characteristics and advantages. Because of the powerful self-healing capabilities and smart grid has good compatibility, also impose considerable demands on their computing model, cloud computing technology in the smart grid information platform construction in the role will fully exposed. Cloud computing as an emerging computing model, which not only can form a powerful computing and storage platforms, but also to achieve dynamic expansion through the integration of a variety of com- puting devices, greatly improving the computing power and storage capacity. Meanwhile, the benefits of virtualization cloud computing greatly improving the utilization of system resources, but also because each computing platform for effective integration advantages, enhance economies of scale power companies, provide an important and effective collaboration for the safe operation of power systems of protection.

Amazon, Google, IBM and other companies were made "cloud Plan", such as Google's Blue Cloud, Amazon AWS, are cloud computing make some contribution, academia conduct some research in order to promote the popularity of cloud computing. Such as Carnegie Mellon University launched a cloud-based smart grid Hadoop platform and the project on the Tennessee Valley Authority, provide a platform for power grids and other data processing, highlighting the Hadoop cheap and reliable, and other advantages. In addition, as early as 2008, IBM Science and Education Industrial Park in Wuxi established the first cloud computing center of our local, is the world's first commercial cloud computing center operations. In 2014, Alibaba Group has also successfully established a cloud computing platform for a number of domestic software companies to provide storage and computing resources (Ruifeng Zhao, 2013). 
3 THE MAIN PROBLEM IS STILL THE SMART GRID INFORMATION PLATFORM FACING

The transition to a cloud computing data center. Our data center because of the different regions and the degree of information presents different characteristics, some data centers already have a basic cloud computing capabilities, but most data centers still remain in the initial level, to ensure the normal operation of the existing data center power under the premise, gradual transition to cloud computing data center in order to meet future requirements of the development of smart grid.

Restricting data transmission problem. State Grid Corporation between provincial branches and there are a lot of data needs to be transmitted, will bring massive data transmission efficiency decreases, taking into account the load balancing device between the cloud node, thus building a wide area network channels to resolve data transmission problem.

Security of data transmission. Data Center for Cloud Computing power companies to build internal private cloud, capable of self-maintenance and management, system wide area network data transmission services, according to the third security zone defined security partition, and install proprietary equipment production area and external public network isolation .

\section{CLOUD COMPUTING APPLICATIONS IN THE SMART GRID INFORMATION POLICY PLATFORM}

Integrate and optimize the management of heterogeneous resources. Among the smart grid systems, data resources, information systems between various platforms and mutually dispersed, severe heterogeneity, thus leading to the integration of the quality of information sharing to increase difficulty, unable to effectively improve network management efficiency for the power distribution system electrical work quality management and marketing operations system maintenance upgrade will not be able to effectively meet demand (Jie Yang, 2014). Cloud computing technology enables the power system to effectively integrate the various business data and computing resources on the basis of information for business collaboration platform built on effective, able to satisfy the huge demand for smart grid information resource sharing. Cloud computing network virtualization and application virtualization technology, the server virtualization technology and other resources to achieve each service abstraction, and then realize the difference between the system and the software effectively eliminated by the appropriate method to achieve the effective integration of information resources, and then to optimize the management, greatly improving the efficiency of heterogeneous resources management applications.
Distributed storage and management of large volume of data. After application of smart grid information platform construction, customer service requests varied, huge data volume information brought it to the storage and management of data provides a great challenge. Cloud computing technology in general face amount of data each smart grid system and unit collected using forms distributed storage of data to achieve the effective storage, greatly to ensure the security and reliability of data storage. GFS effective use of technology with each node, the master server is responsible for the effective management of the whole file system, the file system and complete the work of the metadata stored in the data block is the main server for data storage block sliced, and by saving three or more redundant backup storage. At the same time, client access and complete access to the main block server information.

Fast Power System Parallel Computation and Treatment. Smart grids and information platform for the large volume of data to provide stable operation of the power grid data base, but more critical is the analysis and design of each system operating data, planning and decision-making, and even failures and transient stability, state estimation and intelligent a variety of computing and content analysis and decision-making process, its huge computing needs, general computing system can not reach its goal (Wang, Mengxue, 2015). Cloud computing technology through parallel computing technology has greatly enhanced parallel processing capability of the power system, which provides parallel programming model makes more simple and efficient parallel algorithms. MapReduce as Google made an important parallel programming model, and its role in large data sets in parallel computing plays a very prominent. Parallel processing job results by the client to achieve effective user submitted, via decomposition automated master role, the corresponding task scheduling to working nodes and nodes work completed by the specific calculations and perform the appropriate task.

\section{SMART GRID INFORMATION PLATFORM CLOUD COMPUTING ANALYSIS}

Cloud Computing Smart Grid Information Platform Architecture. Smart grid technology information platform architecture cloud computing include the following several levels and aspects (Yan Cui, 2015): First, that the platform infrastructure layer is the integration of the various hardware resources and related management capabilities of virtualized using virtual technology to achieve the corresponding hardware devices such as computers and network storage devices abstraction, and then complete the automation and optimization of resource data and load management; secondly, the main platform for the integration of the various layers of software 
resources to achieve effective optimization cloud middleware to meet electricity business application security and usability for the huge demand; in addition, the business application layer is the effective integration of the various cloud applications, and its main role is to power enterprise management and marketing, and business intelligence market trading operations analysis.

Design Smart Grid Condition Monitoring Cloud Computing Platform. Design Cloud Computing smart grid condition monitoring should be integrated cloud computing platform that the platform architecture. Considering the smart grid is an important national infrastructure, and condition monitoring is different from search engines and other typical Internet applications, Google's GFS and BigTable, Amazon's EC2, as well as Microsoft's Windows Azure cloud computing technology can not be directly applied, should be used Hadoop technology as applied technology, using HBase as a mass data management system, using the Hadoop distributed file system (HDFS) as a distributed storage system, while Xen as a virtual machine. Hadoop master / slave architecture, the data split into a plurality of data blocks are stored on different storage nodes.

\section{CONCLUSION:}

Cloud computing technology as a new and efficient method of calculation, the application with the smart grid information platform construction, can greatly improve the efficiency of the operation and management platform, and lay a solid foundation for the security and stability of power system operation. Combined with the smart grid condition monitoring features, the development of special solutions on the basis of the actual situation of the power system in order to give full play to the advantages of the smart grid information platform, providing huge security operation for the management and maintenance of the Smart Grid to achieve the rapid development of power enterprises.

\section{REFERENCES:}

Jie Yang, Qian Nie, Yi Yang, the effect of the application of cloud computing platform based on smart grid information [J] Electronics and Software Engineering, 2014,22: 189-190.

Ruifeng Zhao, Jian-gang LU smart grid cloud computing platform [J]. GUIDE software, 2013,12: 41-43.

Wang, Mengxue He of cloud computing driving force for the smart grid Ping [J] Chinese New Telecommunications, 2015,07: 9.

Yan Cui cloud computing research smart grid information platform [J] based on digital technology and applications, 2015,05: 103.

Zhiyong Xu cloud-based smart grid information platform [J] Science and Technology Communication, 2013,23: $204+$ 135. 